\title{
Preflare chromospheric and photospheric line-of-sight velocities
}

\author{
K. V. Alikaeva ${ }^{1} \dagger$ and S. N. Chornogor ${ }^{1}$ \\ ${ }^{1}$ Main Astronomical Observatory, National Academy of Sciences of Ukraine, \\ email: alikaeva@mao.kiev.ua, chornog@mao.kiev.ua
}

\begin{abstract}
The preflare plage spectra were obtained with the ATsU-26 solar telescope mounted at the Peak Terskol. The spectra were acquired from $1 \mathrm{~h}$ to $20 \mathrm{~min}$ before the flare onset. The most prominent peculiarity of all the observed events are the tilted $H_{\alpha}$-emission details which appear less than 40 min before the flare. This result may testify to spiral structure of the ascending $H_{\alpha}$ loops. The ascending velocities in the chromosphere attain more than $10 \mathrm{~km} / \mathrm{s}$. Two crossing $H_{\alpha}$ loops were observed to interact before $1 \mathrm{n}$ flare. As a result of their interaction, the redistribution of velocities occurs in the both chromosphere and photosphere. This can be a consequence of the preflare reconnection at the lower atmospheric layers. As the flare onset approaches the ascend of photospheric material start to dominate. The preflare line-of-sight velocities exceed considerably those in the neighbouring unperturbed regions.
\end{abstract}

In this study we examine the line-of-sight (LOS) velocity field at the photospheric and chromospheric levels at the sites of future flares in less than 1 hour before the flares of different power. The spectra were acquired at the solar telescope of the High-Altitude Terskol Station of the Main Astronomical Observatory (Ukraine).

The line-of-sight velocities were measured from the Doppler shifts of solar lines with respect to several neighboring telluric lines. They were determined for various moments of flare evolution and for various photometric cross-sections with corrections for the Earth's rotation about its axis and revolution around the Sun, for the rotation of the Sun, and for the gravitational shift. The rms error $\sigma$ of the velocity did not exceed $200 \mathrm{~m} / \mathrm{s}$ for the chromospheric motions from the $H_{\alpha}$ line and $100 \mathrm{~m} / \mathrm{s}$ for the photospheric motions from absorption lines.

The interesting peculiarities have been revealed: a) the short-term X-ray spikes (GOES data) precede the flares without any manifestations in the $H_{\alpha}$ emission. It will be noticed that the greater power of the following flare the more intensive preflare spikes; b) the tilted emission details in $H_{\alpha}$ spectra (see Figure 1) appeared in less than 40 min before the flare onset (on earlier stage they are absent). Our observations show that such $H_{\alpha}$ spectra can appear before the flares when the twisted magnetic loops ascend from the sub-photosphere.

As an example we represent the result of the LOS velocity field determination in the chromosphere and photosphere before 1n/M1.0 flare on 26 August 1990. The tilted pattern is peculiar not only to $H_{\alpha}$-intensities, but also LOS velocities (see Figure 2). In the spectrogrammes $H_{\alpha}$-line emission was observed as two crossing tilted details, which correspond to $H_{\alpha}$-loops - L1 and L2. It is interesting, that redistribution of chromospheric velocities in these loops occurs at the preflare stage. Figure 2 (left and middle panels) shows the decreasing of ascending motions in the loop L1 and the increasing of descending motions in the loop L2 as the flare onset approaches.

$\dagger$ Present address: Main Astron. Observ., 27 Akademika Zabolotnoho St., 03680 Kyiv, Ukraine. 


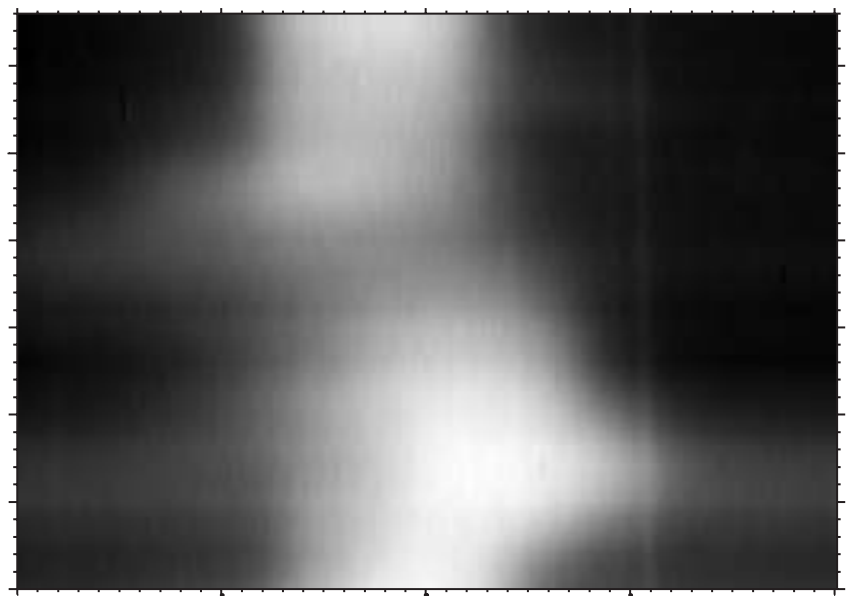

Figure 1. Tilted $H_{\alpha}$-spectra observed before the flare of 26 August 1990.
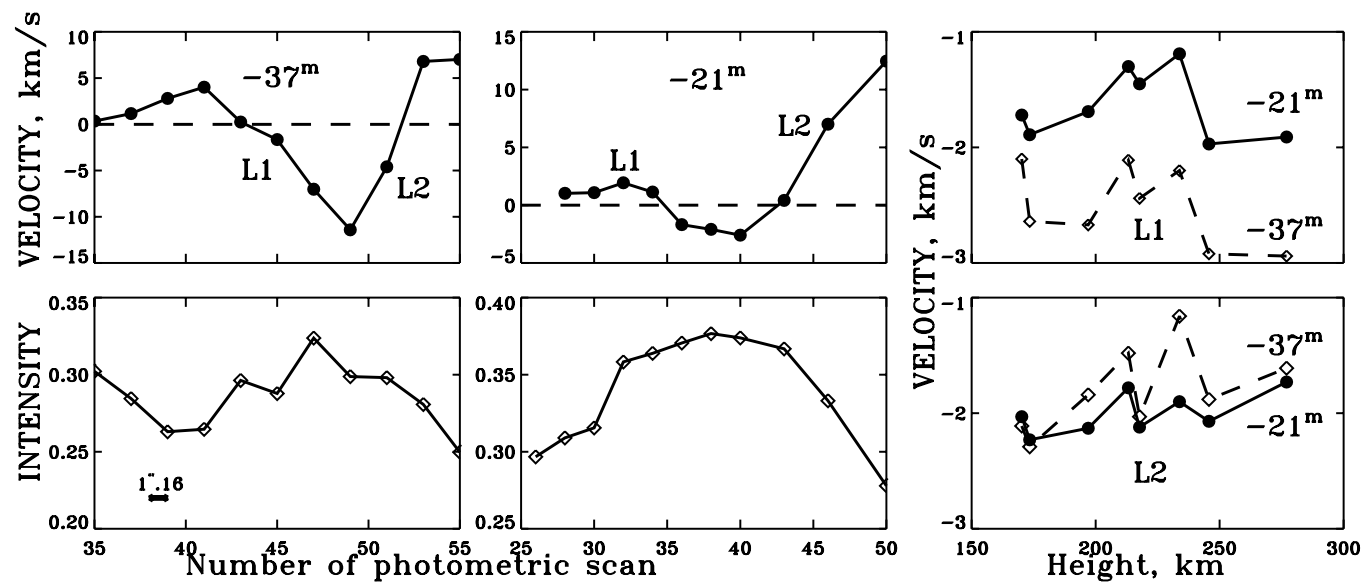

Figure 2. LOS velocity (left panel) and intensity at the $H_{\alpha}$ line center (middle panel) for different photometric scans and height distributions of LOS velocities in the photosphere (right panel) for two moments before 1n/M1.0 flare onset.

The similar process take place at the photospheric levels also. Figure 2 (right panel) shows the height distributions of LOS velocities in the photospheric parts of loops L1 and L2. Thus, the velocity variations at the chromospheric and photospheric levels before $1 \mathrm{n}$ flare may to testify to the probability of the reconnection at the lower atmospheric layers in the preflare stage.

As may be seen from the our data on LOS velocities for three more faint flares the ascent of matter predominates in the both chromosphere and photosphere at the preflare stage. The height interval of ascending motions in the photosphere expands and velocities increase as the flare onset approaches. The LOS velocity values exceed considerably those in the neighbouring undisturbed regions in all the events under investigation.

The peculiarities of the velocity field can be an experimental confirmation of an important role of the photosphere at the preflare stage. The photosphere should be considered as a connecting link between processes in the subphotospheric level and the following flare manifestations in the chromosphere and corona. 